\title{
SEPSE: UMA EVOLUÇÃO DE CONCEITOS
}

\section{ARTIGO DE REVISÃO}

MATHIAS, Thalita Thauana Pistori Alencar ${ }^{1}$

SOUZA, Douglas Silveira Rodrigues de ${ }^{2}$

IKEDA, Camila Sekine ${ }^{3}$

SILVA, Dieison Pedro Tomaz da ${ }^{4}$

MATHIAS, Thalita Thauana Pistori Alencar. Et. al. Sepse: uma evolução de conceitos. Revista Científica Multidisciplinar Núcleo do Conhecimento. Ano 04, Ed. 03, Vol. 03, pp. 32-46. Março de 2019. ISSN: 2448-0959.

\section{RESUMO}

Estima-se, segundo o Conselho Federal de Medicina (2015), que 600 mil novos casos de sepse ocorrem a cada ano no Brasil, com custo de US\$9,6mil por paciente. Acredita-se que a alta taxa de incidência se deve ao prolongamento da expectativa de vida da população, as novas tecnologias na área da saúde e pelo aumento de pacientes imunocomprometidos, que são os grupos mais suscetíveis ao problema em

\footnotetext{
${ }^{1}$ Acadêmica de Medicina da Universidade Brasil.

${ }^{2}$ Acadêmico de Medicina da Universidade Brasil.

${ }^{3}$ Acadêmica de Medicina da Universidade Brasil.

${ }^{4}$ Graduado em Medicina pela Universidade Brasil, residência médica em clínica médica pela Universidade Brasil, Graduado em Farmácia pela Universidade Federal de Mato Grosso do Sul, docente nos módulos de bases do diagnóstico clínico e bases das terapêuticas e preceptor da disciplina de urgência da graduação em medicina na Universidade Brasil, Médico e docente na graduação de Medicina da Universidade Brasil.
} 
questão (Conselho Federal de Medicina, 2015).Em 1991 foi elaborado o primeiro consenso sobre sepse e choque séptico, desde então surgiram outros consensos como os de 2001, 2014 e 2016. OBJETIVO: O referido artigo visa atualizar o leitor sobre a evolução de conceitos referentes aos consensos de sepse de 1991, 2001 e de 2016. METODOLOGIA: Revisão narrativa da literatura com estratégia de busca definida em artigos nas bases de dados Scientific Electronic Library Online (SciELO) e na U. S. National Library of Medicine (PubMed) e revisão dos consensos SEPSIS1, SEPSIS-2 e SEPSIS-3. CONCLUSÃO: Apesar dos inúmeros estudos e conceitos que surgiram ao longo do tempo sobre sepse e choque séptico, o que poderia gerar dúvidas referente ao assunto em tela, todos foram de suma importância para a construção do saber, para o manejo clínico e para romper paradigmas.

Palavras Chaves: Sepse, Choque Séptico, Unidades de terapia intensiva.

\section{INTRODUÇÃO}

Estima-se, segundo o Conselho Federal de Medicina (2015), que 600 mil novos casos de sepse ocorrem a cada ano no Brasil, com custo de US\$9,6 mil por paciente. A sepse tem altas taxas de mortalidade, com maior tempo de internação e pacientes mais graves, conforme estudo de SALES, J. J. et al. (2006) que analisou dados de 75 UTIs de 65 hospitais do país no qual a taxa de mortalidade para sepse foi de $16,7 \%$.

Acredita-se que a alta taxa de incidência se deve ao prolongamento da expectativa de vida da população, as novas tecnologias na área da saúde e pelo aumento de pacientes imunocomprometidos, que são os grupos mais suscetíveis ao problema em questão (CONSELHO FEDERAL DE MEDICINA, 2015).

Em 1991 foi elaborado o primeiro consenso sobre sepse e choque séptico durante uma conferência, desde então houve eventos semelhantes em 2001 e 2014. Em 2016 o Journal of the American Medical Association (JAMA) publicou o Terceiro Consenso internacional de Definições para Sepse e choque Séptico, que foi denominado como SEPSIS-3, e designou os termos SEPSIS-1 para o que foi previsto em 1991 e SEPSIS-2 para o consenso de 2001 (PLEVIN, R. ET AL., 2017). 


\section{METODOLOGIA}

Revisão narrativa da literatura com estratégia de busca definida em artigos nas bases de dados Scientific Electronic Library Online (SciELO) e na U. S. National Library of Medicine (PubMed) e revisão dos consensos SEPSIS-1, SEPSIS-2 e SEPSIS-3.

\section{DISCUSSÃO}

\subsection{SEPSE, SIRS, SOFA SCORE E QSOFA}

Em 1991, convencionou-se que o sepse era um termo genérico o qual se referia a resposta inflamatória sistêmica à infecção. No ano seguinte, foi introduzido o termo "síndrome da resposta inflamatória sistêmica" (SIRS), que é considerada na presença de dois ou mais dos seguintes critérios:

- Taquicardia (frequência cardíaca > 90/min)

- Taquipneia (frequência respiratória $>20 / \mathrm{min}$ ou $\mathrm{PaCO}_{2}>32 \mathrm{mmHg}$ )

- Leucocitose (leucócitos $>12000 / \mu \mathrm{L}$ ) ou leucopenia $(<4000 / \mu \mathrm{L})$ ou mais que $10 \%$ de bastonetes

- Hipertermia $\left(>38^{\circ} \mathrm{C}\right)$ ou hipotermia $\left(<36^{\circ} \mathrm{C}\right)$.

Devido ao grande uso na prática, e por ter servido como critério de base em diversos trabalhos do período, em 2001 o termo "sepse" foi definido como a presença de SIRS somada a infecção e "sepse grave" como sepse associada a disfunção orgânica, hipoperfusão ou hipotensão. Ainda neste contexto, as definições de SIRS apresentavam limitações e era muito importante que houvessem ferramentas para diagnosticar sepse, então foi elaborada uma lista de possíveis achados de resposta inflamatória sistêmica à infecção (tabela 01) que auxiliariam na caracterização do paciente possivelmente séptico e que continuaram a serem utilizados nas diretrizes de 2012 (LEVY, Mitchell M. et al., 2001) (DELLINGER, R. Phillip et al 2007). 
Segundo as Diretrizes internacionais para tratamento de sepse grave e choque séptico (2012), sepse é entendida como a presença presumida ou comprovada de infecção com manifestações sistêmicas dessa e sepse grave manteve a definição anterior.

Tabela 01 Critérios Diagnósticos para Sepse

\begin{tabular}{|c|c|}
\hline \multirow[t]{6}{*}{ Critérios gerais } & Taquipneia (> 20 irpm) \\
\hline & Febre $>38,3^{\circ} \mathrm{C}$ ou Hipotermia $<36^{\circ} \mathrm{C}$ \\
\hline & Estado Mental Basal Alterado \\
\hline & Edema ou Balanço Hídrico Positivo \\
\hline & Hiperglicemia na ausência de Diabetes (> 140mg/dl) \\
\hline & $\begin{array}{l}\text { Hipotensão, sendo: PAS }<90 \mathrm{mmHg} \text { ou PAM }<70 \mathrm{mmHg} \text { ou } \\
\text { Redução da PAS }>40 \mathrm{mmHg} \text { em adultos ou menos de } 2 \\
\text { desvios padrões abaixo para a idade }\end{array}$ \\
\hline \multirow{2}{*}{$\begin{array}{l}\text { Critérios } \\
\text { Inflamatórios }\end{array}$} & Leucocitose $>12.000 / \mathrm{mcL}$ ou Leucopenia $<4.000 / \mathrm{mcL}$ \\
\hline & $\begin{array}{l}\text { Contagem de leucócitos normais com mais de } 10 \% \text { de } \\
\text { formas jovens } \\
\text { Dosagem sérica de Proteína C Reativa }>2 \text { desvios padrões } \\
\text { acima do normal } \\
\text { Dosagem séria de procalcitonina } 2 \text { desvios padrões acima } \\
\text { do normal }\end{array}$ \\
\hline \multirow{2}{*}{$\begin{array}{ll}\text { Critérios } & \text { de } \\
\text { Perfusão } & \end{array}$} & Lactato sérico > 1mmol/L \\
\hline & Redução do enchimento capilar \\
\hline \multirow{7}{*}{$\begin{array}{l}\text { Critérios } \\
\text { Disfunção } \\
\text { Orgânica }\end{array}$} & Hipoxemia $\left(\mathrm{PaO}_{2} / \mathrm{FiO}_{2}<300\right)$ \\
\hline & Aumento da Creatinina sérica $>0,5 \mathrm{mg} / \mathrm{dl}$ \\
\hline & Oligúria aguda (diurese $<0,5 \mathrm{ml} / \mathrm{Kg} / \mathrm{h}$ ) \\
\hline & Ausência de Ruídos Hidroaéreos \\
\hline & Bilirrubina Total sérica $>4 \mathrm{mg} / \mathrm{dl}$ \\
\hline & INR $>1,5$ ou TTPA $>60$ s \\
\hline & Plaquetas $<100.000 / \mathrm{mcL}$ \\
\hline
\end{tabular}


Fonte: DELLINGER, R. P., et al., 2007

Sepse, como foi definida em 2016, é uma disfunção orgânica possivelmente letal causada por uma resposta inadequada do hospedeiro a uma infecção. Por representar um mecanismo fisiopatológico mais complexo que somente o conceito de uma infecção somada a resposta inflamatória, o termo sepse grave foi abolido do terceiro consenso por ter se tornado desnecessário. (SINGER, M. et al., 2016)

O consenso SEPSIS-3 decidiu que dois critérios de SIRS acompanhado de infecção não era apropriado para distinguir entre uma resposta inflamatória fisiológica à alguma injúria e sepse (PLEVIN, R. 2017). Dessa forma, a necessidade da presença de SIRS para diagnosticar sepse foi excluída e o foco foi então alterado para a identificação de disfunção e falência de órgãos, em conformidade com a nova definição.

Por ser mais conhecido e mais simples, o score Sequencial Organ Failure Assessment (SOFA) (tabela 02) foi a escolha para avaliar o grau de disfunção orgânica em pacientes com infecção. Pressupõe-se que a pontuação basal de um paciente sem uma disfunção preexistente é igual a 0 (zero), dessa forma uma pontuação SOFA $\geq 2$ indica risco de evolução do quadro para morte (SINGER, M. et al., 2016) e, em comparação com o SEPSIS-2, as definições do terceiro consenso tiveram maior valor preditivo de morte (POUTSIAKA, D. D. et al., 2017).

Embora ter sido considerada uma boa ferramenta para caracterizar clinicamente um paciente séptico, o score SOFA não é indicado para guiar o manejo dos casos. Outra crítica para o uso do score é que 4 (quatro) dos seus critérios são obtidos de análises laboratoriais, um deles é uma resposta ao tratamento e apenas a escala de coma de Glasgow seria um critério clínico, tornando difícil sua aplicação fora de uma unidade de terapia intensiva. Vale ressaltar que o SOFA score não é conceito novo no contexto de sepse, uma vez que no SEPSIS-2 já havia sido recomendado para auxiliar na avaliação de pacientes sépticos graves. (CARNEIRO, A. H., ANDRADE-GOMES, J., PÓVOA, P., 2016) (LEVY, Mitchell M. et al., 2001) 
Para oferecer uma ferramenta simples para identificação de pacientes com sepse, foi também proposto um sistema de pontuação denominado "quickSOFA" score (qSOFA) que tem como parâmetros frequência respiratória $\geq 22$, Alteração no nível de consciência (Escala de Coma de Glasgow $<15$ ) e pressão arterial sistólica $\leq 100 \mathrm{mmHg}$ e que indica risco elevado de evolução para um quadro séptico se dois ou mais critérios estiverem presentes.

Apesar de o qSOFA possuir valor preditivo similar ao SOFA, é importante ressaltar que sozinho ele não define sepse e não substitui uma boa avaliação clínica e laboratorial, mas pode ser um bom preditor para piora clínica. Os benefícios do uso desse sistema de pontuação são a possibilidade de uma avaliação rápida e repetida e de não serem necessários nenhum teste laboratorial. (SINGER, M. et al., 2016) (PLEVIN, R. et al., 2017)

\section{Tabela 02. Sequential [Sepsis-Related] Organ Failure Assessment Score}

Sistema: Pontuação:

\begin{tabular}{|c|c|c|c|c|c|}
\hline & 0 & 1 & 2 & 3 & 4 \\
\hline \multicolumn{6}{|l|}{ Respiratório: } \\
\hline $\begin{array}{l}\mathrm{PaO} 2 / \mathrm{FIO} 2, \\
\mathrm{mmHg}\end{array}$ & $\geq 400$ & $<400$ & $<300$ & $\begin{array}{l}<200 \quad \text { com } \\
\text { suporte } \\
\text { ventilatório }\end{array}$ & $\begin{array}{l}<100 \quad \text { com } \\
\text { suporte } \\
\text { ventilatório }\end{array}$ \\
\hline \multicolumn{6}{|l|}{ Coagulação: } \\
\hline $\begin{array}{l}\text { Plaquetas, } \\
\times 10^{3} / \mu \mathrm{L}\end{array}$ & $\geq 150$ & $<150$ & $<100$ & $<50$ & $<20$ \\
\hline \multicolumn{6}{|l|}{ Fígado: } \\
\hline $\begin{array}{l}\text { Bilirrubina, } \\
\mathrm{mg} / \mathrm{dL}\end{array}$ & $<1.2$ & $1.2-1.9$ & $2.0-5.9$ & $6.0-11.9$ & $>12.0$ \\
\hline Cardiovascular & $\begin{array}{l}\mathrm{PAM} \geq 70 \\
\mathrm{~mm} \mathrm{Hg}\end{array}$ & $\begin{array}{l}\text { PAM } \\
<70 \\
\mathrm{~mm} \mathrm{Hg}\end{array}$ & $\begin{array}{l}\text { Dopamina } \\
<5 \quad \text { ou } \\
\text { dobutamina }\end{array}$ & $\begin{array}{l}\text { Dopamina } 5,1- \\
15 \text { ou } \\
\text { epinefrina } \leq 0,1 \\
\text { ou }\end{array}$ & $\begin{array}{l}\text { Dopamina } \\
>15 \text { ou } \\
\text { epinefrina } \\
>0,1 \quad \text { ou }\end{array}$ \\
\hline
\end{tabular}

Disponível em: https://www.nucleodoconhecimento.com.br/saude/sepse-uma-evolucao-de- 


\begin{tabular}{|c|c|c|c|c|c|}
\hline & & & $\begin{array}{l}\text { (qualquer } \\
\text { dosagem) }\end{array}$ & $\begin{array}{l}\text { norepinefrina } \\
\leq 0,1\end{array}$ & $\begin{array}{l}\text { norepinefrina } \\
>0,1\end{array}$ \\
\hline $\begin{array}{l}\text { Sistema } \\
\text { Nervoso } \\
\text { Central: }\end{array}$ & & & & & \\
\hline $\begin{array}{l}\text { Escala de coma } \\
\text { de Glasgow }\end{array}$ & 15 & $13-14$ & $10-12$ & $6-9$ & $<6$ \\
\hline Renal: & & & & & \\
\hline $\begin{array}{l}\text { Creatinina, } \\
\mathrm{mg} / \mathrm{dL}\end{array}$ & $<1.2$ & $1.2-1.9$ & $2.0-3.4$ & $3.5-4.9$ & $>5.0$ \\
\hline $\begin{array}{l}\text { Débito Urinário, } \\
\mathrm{mL}\end{array}$ & & & & $<500$ & $<200$ \\
\hline
\end{tabular}

\subsection{CHOQUE SÉPTICO}

Nas duas primeiras diretrizes choque séptico foi definido como um estado agudo de falência circulatória causado por hipotensão (presença de, pelo menos, um dos parâmetros: PAS < 90mmHg/PAM < 70mmHg; decréscimo maior que $40 \mathrm{mmHg}$ na PAS ou maior que dois desvios padrões do padrão de normalidade para a idade) refratária a terapia volêmica adequada, que não pode ser explicada por outras causas. (DELLINGER, R Phillip et al 2007)

Em 2016 choque séptico é descrito como um evento subsequente à sepse que causa alterações metabólicas e circulatórias pronunciadas levando ao aumento da mortalidade. Para elaborar uma definição e critérios clínicos, foi realizada uma revisão sistemática de evidência, a qual elegeu 3 variáveis (hipotensão, níveis elevados de lactato sérico e necessidade de terapia vasopressora) que são importantes no processo de identificação do quadro (SINGER, M. et al., 2016). 
Baseando-se nos 3 (três) critérios, pacientes com choque séptico podem ser identificados por hipotensão persistente que requer terapia vasopressora para manutenção de valores $\geq 65 \mathrm{mmHg}$ e que possuam valores de lactato sérico $>2 \mathrm{mmol} / \mathrm{L}$ $(18 \mathrm{mg} / \mathrm{dL})$ mesmo recebendo terapia de reposição volêmica adequada (SINGER, M. et al., 2016).

\subsection{DIRETRIZES PARA GESTÃO DE CASOS, TERAPIA INICIAL DIRIGIDA POR OBJETIVOS (EGDT) E OS PACOTES DE CUIDADOS DA CAMPANHA SOBREVIVENDO À SEPSE (SSC)}

Pode-se dizer que o primeiro grande trabalho que sugeria uma abordagem para o paciente séptico ou em choque séptico foi publicada em 2001. Nesse trabalho, que avaliou 288 pacientes, Rivers et al. (2001) concluiu que o uso da EGDT proporcionava benefícios significativos nos pacientes, que apresentaram uma taxa de mortalidade de $30,5 \%$ enquanto com o grupo que recebera terapia padrão apresentou mortalidade de 46,5\%. Entretanto, estudos que buscavam avaliar os benefícios da EGDT publicados nos anos seguintes, falharam em evidenciar benefícios de seu uso e apesar de não ser capaz de piorar desfechos, a EDGT foi também associada a custos elevados de operação. (PLEVIN, R. et al., 2017)

Dessa forma, em 2004, com as evidências disponíveis na época, foram publicadas as primeiras diretrizes da SSC que posteriormente foram revisadas em 2008, e novamente em 2012. Em 2016 foram publicadas as Diretrizes Internacionais para a gestão de sepse e choque séptico: 2016, no Critical Care Medicine Journal. (RHODES, A. et al., 2017)

Concomitante as publicações das diretrizes, a SSC vem também elaborando os "pacotes de sepse", que são desenvolvidos separadamente das diretrizes e de modo que possam ser atualizados em vista de novas evidências. Anterior à publicação das diretrizes de 2016 haviam dois pacotes, o "pacote de 3 horas" e o "pacote de 6 horas", com a publicação das últimas diretrizes foi elaborado o "pacote de 1 hora", que é a 
revisão e combinação dos dois pacotes anteriores e propõe o início imediato da ressuscitação e do manejo dos pacientes. (LEVY, M. M., et al. 2018)

\section{Pacote de cuidados de 1-hora da SSC}

Mensurar níveis de lactato. Repetir se o lactato inicial for $>2 \mathrm{mmol} / \mathrm{L}$

Coletar culturas sanguíneas antes de iniciar antibioticoterapia

Administrar antibióticos de amplo espectro

Iniciar rapidamente a administração de $30 \mathrm{ml} / \mathrm{kg}$ de cristaloides para corrigir hipotensão ou lactato $\geq 4 \mathrm{mmol} / \mathrm{L}$

Administrar vasopressores se o paciente está hipotenso durante ou após a ressuscitação volêmica inicial para manutenção de uma MAP $\geq 65 \mathrm{mmHg}$ Fonte: LEVY, M. M., et al., 2018

\subsection{RESSUSCITAÇÃO INICIAL}

As diretrizes de 2012, por Dellinger, R. P. et al. (2012), recomendam ressuscitação protocolizada e quantitativa dos pacientes identificados com hipotensão induzida por sepse assim que seja identificada a hipotensão e não devendo ser adiada caso não seja feita admissão imediata na UTI. Metas foram definidas para as primeiras seis horas de tratamento, são elas:

- PVC 8-12 mm Hg

$-P A M \geq 65 \mathrm{~mm} \mathrm{Hg}$

— Diurese $\geq 0.5 \mathrm{~mL} \cdot \mathrm{kg} \cdot \mathrm{hr}$

- Saturação de oxigenação da veia cava superior (Scvo2) ou saturação de oxigênio venoso misto (SvO2) de $70 \%$ ou $65 \%$, respectivamente.

Em 2016, na publicação de Rhodes, et al. (2017), recomendação é de iniciar tratamento e ressuscitação o mais rápido possível. Nas 3 primeiras horas deve-se iniciar ressuscitação da hipoperfusão induzida por sepse com pelo menos $30 \mathrm{ml} / \mathrm{kg}$ de

Disponível em: https://www.nucleodoconhecimento.com.br/saude/sepse-uma-evolucao-de- 
cristaloide intravenoso que deve ser seguida por reavaliação frequente do estado hemodinâmico que guiará a administração adicional de fluidos, se necessária. (RHODES, A. et al., 2017)

A reavaliação deve incluir exame clínico completo e avaliação das variáveis fisiológicas e monitoramentos invasivos ou não, conforme estiverem disponíveis. Caso o diagnóstico não esteja claro, recomenda-se avaliação hemodinâmica para determinar o tipo de choque. (RHODES, A. et al., 2017)

A manutenção da MAP com valores de pelo menos $65 \mathrm{mmHg}$ se mantêm para pacientes em choque séptico que necessitem de vasopressores e continua como sugestão realizar a reanimação orientada para normalizar os níveis de lactato caso estejam elevados. (DELLINGER, R. P. et al., 2012) (RHODES, A. et al., 2017)

\subsection{TRIAGEM PARA SEPSE E MELHORIA DE DESEMPENHO}

A SSC recomenda que seja feita triagem em pacientes com doenças graves a fim de diagnosticar sepse e dar início ao tratamento precocemente. O diagnóstico precoce e a implementação imediata de terapias apresentam melhores resultados e redução da mortalidade. (DELLINGER, R. P. et al., 2012) (RHODES, A. et al., 2017)

Destaca-se que uma equipe multidisciplinar deve fazer parte dos programas de melhoria de desempenho, que devem incluir desenvolvimento e implementação de protocolo, indicadores segmentados, coleta de dados constante para que possam ser avaliados e a partir deles haja um feedback. (DELLINGER, R. P. et al., 2012) (RHODES, A. et al., 2017)

A implementação dos pacotes de recomendações e diretrizes elaborados pela Campanha de Sobrevivência à Sepse (CSS) podem proporcionar melhoria na qualidade da terapia de sepse e tem servido como base para os programas de melhoria de desempenho. (DELLINGER, R. P. et al., 2012) (RHODES, A. et al., 2017) 


\subsection{DIAGNÓSTICO}

A recomendação permanece obter culturas adequadas, que incluem pelo menos dois conjuntos de hemoculturas (aeróbicas e anaeróbicas), antes de iniciar antibioticoterapia em pacientes com suspeita de sepse ou choque séptico, desde que isso não atrase o início dela. Os sítios de coleta recomendados são pelo menos um por via percutânea e uma amostra de cada dispositivo de acesso vascular que esteja instalado a mais de 48 horas, além de culturas de outros locais que sugerem ser a fonte da infecção.(DELLINGER, R. P. et al., 2012) (RHODES, A. et al., 2017)

\subsection{ANTIBIOTICOTERAPIA}

Em 2016 recomenda-se iniciar a administração de antibióticos o mais rápido possível e dentro de 1 hora da identificação do quadro séptico ou choque séptico. A escolha da terapia deve ser empírica, com um ou mais medicamentos que cubram todos os prováveis patógenos (inclusive fungos ou vírus). Diferente de 2012 que recomendava tratar sepse neutropenia/bacteremia com terapia antimicrobiana combinada empírica, 2016 não recomenda terapia combinada para tratamento rotineiro nesses casos. (Dellinger, R. P. et al., 2012) (Rhodes, A. et al., 2017)

Quando identificado o patógeno e suas sensibilidades ou quando seja notada melhora clínica, recomenda-se adequar a terapia aos achados ou descontinua-la a fim de evitar toxicidade, resistência antimicrobiana e reduzir custos. Pacientes com estados inflamatórios graves que sugerem origem não infecciosa não devem receber antimicrobianos. (Dellinger, R. P. et al., 2012) (Rhodes, A. et al., 2017)

As diretrizes de 2012 sugeriam que o tratamento empírico de sepse grave não ultrapassasse 3-5 dias, (Dellinger, R. P. et al., 2012) já 2016 traz que a terapia combinada, se utilizada para tratar choque séptico, seja ela direcionada ou empírica, deve ser descontinuada logo nos primeiros dias ao sinal de melhora clínica ou resolução da infecção (Rhodes, A. et al., 2017). O tempo de tratamento sugerido é de 7-10 dias para infecções mais graves associadas com sepse e choque séptico, 
entretanto cursos mais longos de tratamento podem ser adequados para pacientes com uma resposta clínica lentificada. (Dellinger, R. P. et al., 2012) (Rhodes, A. et al., 2017)

Ambas diretrizes recomendam avaliação diária dos casos para redução da terapia antimicrobiana, a fim de evitar toxicidade, resistência bacteriana aos antibióticos e reduzir custos. A redução da antibioticoterapia ou descontinuação pode ser suportada através da avaliação dos níveis de procalcitonina. (Dellinger, R. P. et al., 2012) (Rhodes, A. et al., 2017)

\subsection{CONTROLE DE ORIGEM}

Substancialmente as recomendações se mantiveram. Recomenda-se realizar diagnostico anatômico específico de infecção, e se possível, a intervenção deve acontecer o mais rápido possível. Caso algum dispositivo de acesso vascular seja a possível fonte de infecção, eles devem ser removidos rapidamente após o estabelecimento de outro acesso. Entretanto, em 2016 não há mais a sugestão sobre a maneira de intervir em caso de necrose peri pancreática e o uso de técnicas menos invasivas possíveis para conseguir executar o controle de origem. (Dellinger, R. P. et al., 2012) (Rhodes, A. et al., 2017)

\subsection{TERAPIA DE FLUIDOS}

Cristaloides são os fluidos de escolha para ressuscitação inicial em ambas as diretrizes, mas em 2016 cristaloides balanceados e soro fisiológico também são sugeridos como opção para ressuscitação liquida de pacientes com sepse e choque séptico. Além de cristaloides, albumina também pode ser usada caso os pacientes necessitem de quantidades grandes de cristaloides. $O$ uso de amidos de hidroxietila manteve-se desencorajado. (Dellinger, R. P. et al., 2012) (Rhodes, A. et al., 2017) 


\subsection{MEDICAÇÃO VASOATIVA}

Norepinefrina continua sendo primeira escolha de vasopressor. Dopamina pode ser usada como alternativa em pacientes altamente selecionados e seu uso em baixas doses continua não sendo indicado para proteção renal. Mantém-se a sugestão do uso de até $0,03 \mathrm{U} / \mathrm{min}$ de vasopressina ou adrenalina para aumento da PAM ou diminuição da dose de norepinefrina. (Dellinger, R. P. et al., 2012) (Rhodes, A. et al., 2017)

Dobutamina continua sugerida em casos onde há persistência de hipotensão mesmo com terapia de fluidos e vasopressora adequadas, entretanto em 2016 não há especificação de dose inicial como em 2012. O mesmo acontece com a recomendação do uso de fenilefrina para tratamento de choque séptico em alguns casos específicos, que existia nas diretrizes de 2012, mas não está presente em 2016. (DELLINGER, R. P. et al., 2012) (RHODES, A. et al., 2017)

\subsection{CORTICOESTEROIDE}

Em 2016 as recomendações sobre o uso de corticosteroides foram simplificadas e o que permaneceu foi a sugestão do uso de $200 \mathrm{mg}$ por dia de hidrocortisona em casos onde mesmo com uso de técnicas adequadas de ressuscitação e vasopressão não forem suficientes para restaurar o equilíbrio hemodinâmico. (DELLINGER, R. P. et al., 2012) (RHODES, A. et al., 2017

\subsection{PRODUTOS SANGUÍNEOS}

As diretrizes publicadas em 2016 não trouxeram mudanças expressivas nas recomendações para utilização de produtos sanguíneos. A transfusão de hemácias deve ser feita quando, na ausência de condições atenuantes, a concentração de hemoglobina diminuir para $<7,0 \mathrm{~g} / \mathrm{dL}$ em adultos. Não se recomenda 0 uso de eritropoietina para tratar anemia associada à sepse e plasma fresco congelado só deve ser administrado em pacientes com anormalidades de coagulação sanguínea 
acompanhadas de sangramento ativo ou procedimentos invasivos a serem executados. Transfusão profilática de plaquetas pode ser feita quando as contagens forem $<10.000 / \mathrm{mm}^{3}$ na ausência de hemorragia aparente e $<20.000 / \mathrm{mm}^{3}$ se o paciente tiver um risco significativo de sangramento. Contagens de plaquetas > $50.000 / \mathrm{mm}^{3}$ são recomendadas em caso de cirurgias, sangramentos ou procedimentos invasivos ativos. (DELLINGER, R. P. et al., 2012) (RHODES, A. et al., 2017)

\subsection{IMUNOGLOBULINAS, PURIFICAÇÃO SANGUÍNEA, ANTICOAGULANTES E TERAPIA DE BICARBONATO}

Ambas diretrizes não recomendam nem sugerem o uso de imunoglobulinas, técnicas de purificação sanguínea e anticoagulantes no tratamento e manejo de sepse e choque séptico e sugerem não usar terapia de bicarbonato buscando melhora hemodinâmica ou redução da necessidade de vasopressores em pacientes com acidose lática devido a perfusão com pH $\geq 7,15$. (DELLINGER, R. P. et al., 2012) (RHODES, A. et al., 2017)

\subsection{VENTILAÇÃO MECÂNICA}

Recomenda-se o uso de ventilação mecânica em caso de síndrome do desconforto respiratório agudo (SDRA) induzida por sepse. Para tanto deve-se seguir as recomendações específicas sobre o manejo de casos de SDRA devem ser seguidas. Recomenda-se utilização de baixo volume corrente de $6 \mathrm{ml} / \mathrm{kg}$ de peso corporal, pressões de platô $\leq 30 \mathrm{cmH}_{2} \mathrm{O}$, níveis mais altos de pressão positiva ao final da expiração (PEEP) e realização de manobras de recrutamento de alvéolos quando há hipóxia refratária. (Dellinger, R. P. et al., 2012) (Rhodes, A. et al., 2017)

O uso de máscara não invasiva (NIV) para ventilação era recomendado, por Dellinger, R. P. et al. (2012), em casos seletos, já em 2016 não se faz recomendação alguma sobre o uso de NIV. Outra diferença acontece na recomendação sobre utilização de posição prona, que era sugerida quando houvesse razão $\mathrm{PaO}_{2} / \mathrm{FIO}_{2}<100$ e agora 
passou a ser em casos de razão $\mathrm{PaO}_{2} / \mathrm{FlO}_{2}<150$. (Dellinger, R. P. et al., 2012) (RHODES, A. et al., 2017)

Realizar ensaios respiratórios espontâneos para avaliar possibilidade de descontinuação da ventilação mecânica e aplicar um protocolo de redução da dependência são recomendação de ambas diretrizes, mas diferente de 2012, 2016 não especifica nenhum critério para considerar extubação. $O$ uso de agonistas de $\beta$ 2 continua desencorajado e manteve-se a recomendação de usar estratégia de fluidos conservadora e a sugestão de administrar bloqueadores neuromusculares por $\leq 48$ horas e em pacientes com razão $\mathrm{PaO}_{2} / \mathrm{FIO}_{2}<150$. (DELLINGER, R. P. et al., 2012) (RHODES, A. et al., 2017)

\subsection{SEDAÇÃO E ANALGESIA E CONTROLE GLICÊMICO}

Essencialmente as recomendações para sedação e analgesia e controle glicêmico permaneceram as mesmas. Recomenda-se limitar o uso de sedação em pacientes críticos com ventilação mecânica (DELLINGER, R. P. et al., 2012) (RHODES, A. et al., 2017). Dellinger, R. P. et al. (2012) ainda falava sobre o uso de agentes bloqueadores neuromusculares (NMBAs) em pacientes com ARDS, mas as diretrizes de 2018 não trazem nada específico sobre o tema.

A recomendação para controle dos níveis glicêmicos é iniciar administração de insulina assim que forem obtidas duas medições de nível glicêmico no sangue $>180$ $\mathrm{mg} / \mathrm{dL}$ a fim de manter a glicemia $\leq 180 \mathrm{mg} / \mathrm{dL}$. Até estabilização da taxa de infusão de insulina e dos níveis de glicose sérica, monitorizações dos valores de glicemia devem ocorrer a cada 1-2 horas, e após isso, a cada 4 horas, preferindo-se, se possível, utilização de sangue arterial ao sangue capilar para as medições. (DELLINGER, R. P. et al., 2012) (RHODES, A. et al., 2017)

\subsection{TERAPIA DE SUBSTITUIÇÃO RENAL}

São sugestões de ambas as diretrizes utilizar terapias de substituição renal contínuas ou hemodiálise intermitente em pacientes sépticos com doença renal crônica e

Disponível em: https://www.nucleodoconhecimento.com.br/saude/sepse-uma-evolucao-de- 
terapias contínuas com finalidade de facilitar manutenção do equilíbrio de fluidos dos pacientes (DELLINGER, R. P. et al., 2012) (RHODES, A. et al., 2017). Adicionalmente, Rhodes, A. et al. (2017) sugere que terapias de substituição renal não devem ser utilizadas em pacientes com sepse e lesão renal aguda com o propósito de aumentar a creatinina ou em caso de oligúria sem que haja outras indicações para diálise.

\subsection{PROFILAXIA DE TROMBOEMBOLISMO VENOSO (VTE) E ÚLCERA POR ESTRESSE}

A recomendação manteve-se realizar profilaxia farmacológica de VTE, na ausência de contraindicações para uso dos fármacos, e sempre que possível, combiná-la com profilaxia mecânica. Preferir, quando não houverem contraindicações, heparina de baixo peso molecular (LMHW) à heparina não fracionada (UFH). Quando profilaxia farmacológica não for recomendada, utilizar profilaxia mecânica. (DELLINGER, R. P. et al., 2012) (RHODES, A. et al., 2017)

A profilaxia para úlceras por estresse deve ser feita em pacientes que tenham risco de sangramentos gastrointestinais e na ausência de fatores de risco sugere-se que não seja realizada profilaxia. Dellinger, R. P. et al. (2012) sugeria o uso de inibidores de bomba de prótons em vez de antagonistas do receptor $\mathrm{H}_{2}$, entretanto Rhodes, $\mathrm{A}$. et al. (2017) não faz menciona essa preferência e sugere ambas medicações para profilaxia.

\subsection{NUTRIÇÃO}

Nutrição enteral precoce deve ser iniciada, em pacientes que podem ser alimentados dessa maneira, evitando assim nutrição parenteral precoce exclusiva ou combinada com dieta enteral, jejum completo ou apenas glicose intravenosa. Nos 7 dias iniciais, a sugestão é, se necessário, usar glicose intravenosa e seguir introduzindo alimentos entéricos conforme for tolerado e introduzir uma dieta primária hipocalórica (até 500kcal/ dia, por exemplo). (DELLINGER, R. P. et al., 2012) (RHODES, A. et al., 2017) 
O texto publicado em 2016 ainda dispõe sobre suplementação imunomoduladora da mesma forma que o de 2012, não recomendando seu uso. (DELLINGER, R. P. et al., 2012) (RHODES, A. et al., 2017) Em adição, Rhodes, A. et al. (2017) sugere que não se monitorem volumes gástricos residuais em pacientes graves mas que se realize medição de resíduos gástricos em pacientes com alto risco para aspiração e em casos onde há intolerância à dieta podem ser administrados pro cinéticos ou colocados tubos de dieta pós-pilóricos.

\subsection{OBJETIVOS DE TRATAMENTO}

A CSS recomenda discutir objetivos de tratamento com a família do paciente, o mais rápido possível e dentro de 72 horas da internação na UTI. Os objetivos de tratamento devem ser incorporados ao tratamento, assim como os princípios e práticas de cuidados paliativos, quando for aplicável. (DELLINGER, R. P. et al., 2012) (RHODES, A. et al., 2017)

\section{CONSIDERAÇÕES FINAIS}

Após as definições de 2001 viu-se que elas eram demasiadamente simples para compreender o contexto complexo de sepse e suas formas mais graves. As definições seguintes (Sepsis-2) não foram revolucionarias em relação a primeira, tendo apenas feito uma revisão dos critérios e um maior enfoque na condução dos casos. O Sepsis3 surgiu com grandes alterações para as duas versões passadas. Sendo umas delas a retirada da obrigação da presença de SIRS para definir sepse. A introdução dos scores de avaliação de falência de órgãos (SOFA e qSOFA) teve como finalidade melhorar a acurácia do diagnóstico e ser um melhor preditor positivo.

Ainda há, sem dúvidas, muito a ser discutido e aperfeiçoado sobre o tema. A proposta das novas pontuações seria fornecer um modelo clínico de avaliação mais abrangente que os antecessores, entretanto, as recomendações não são sempre universais nem se aplicam perfeitamente em todas as situações. Apesar dos inúmeros estudos e conceitos que surgiram ao longo do tempo sobre sepse e choque séptico, todos foram 
de suma importância para a construção do saber, para o manejo clínico e para romper paradigmas.

\section{REFERÊNCIAS}

BOECHAT, A. L.et al. Sepse: Diagnóstico e Tratamento. Revista Brasileira de Clínica Médica. São Paulo, 2010 set-out;8(5):420-7

CARNEIRO, A. H.; ANDRADE-GOMES, J.; PÓVOA, P. Cara Sépsis-3, Lamentamos Dizer-Iho, Mas Não Gostamos de Si. Medicina Interna, vol. 23, n. 4, p.56-60, dez, 2016.

DELLINGER, R. P., et al Campanha Sobrevivendo à Sepse: Diretrizes internacionais para a gestão de sepse e choque séptico, 2012. Critical Care Medicine, v. 41, n. 2, fev. 2013

Instituto Latino-Americano para Estudos da Sepse. Sepse: um problema de saúde pública. Brasília: CFM, 2015.

LEVY, M. M., et al. 2001 SCCM/ESICM/ACCP/ATS/SIS International Sepsis Definitions Conference. Intensive Care Medicine, vol. 29, n. 4, p. 530-538, fev., 2003.

LEVY, M. M., Evans, L. E., Rhodes, A. The Surviving Sepsis Campaign Bundle: 2018 Update. Critical Care Medicine, vol. 46, n. 6, p 997-1000, Jun, 2018.

PLEVIN, R.; CALLCUT, R. Update in sepsis guidelines: what is really new?. Trauma Surgery \& Acute Care Open, v. 2, n. 1, p.1-6, set. 2017.

POUTSIAKA, D. D. et al. Comparison of the Sepsis-2 and Sepsis-3 Definitions of Sepsis and Their Ability to Predict Mortality in a Prospective Intensive Care Unit Cohort. Open Forum Infectious Diseases. Vol. 4, p. 602, out, 2017 
RHODES, A. et al., Campanha Sobrevivendo à Sepse: Diretrizes internacionais para a gestão de sepse e choque séptico: 2016. Critical Care Medicine. vol. 45, n. 3, p. 486-556, mar. 2016.

SALES JÚNIOR, J. A.et al. Sepse Brasil: Estudo Epidemiológico da Sepse em Unidades de Terapia Intensiva Brasileiras. Revista Brasileira Terapia Intensiva, v. 18, n. 1, p. 9-17, 2006.

SINGER, M., et al. The Third International Consensus Definitions for Sepsis and Septic Shock (Sepsis-3). JAMA, vol. 315, n. 8, p. 801-810, fev., 2016.

Enviado: Dezembro, 2018.

Aprovado: Março, 2019. 